\title{
Crop Pestilence Detection Using Image Segmentation
}

\author{
Vijay E V ${ }^{\mathbf{1}}$, K Siva Anjaneyulu ${ }^{2}$, G Jagadesh $^{3}$, K Poornima Chandana ${ }^{4}$, K Mohan Karthik ${ }^{\mathbf{5}}$ \\ 1, 2, 3, 4, 5 Dept. of ECE, Gudlavalleru Engineering College, Gudlavalleru \\ 1, 2, 3, 4, 5 Asst, Professor, Gudlavalleru Engineering College \\ E-mail:vargilvijay@gmail.com ${ }^{l}$
}

\begin{abstract}
From the numerous hundreds of years, people and other living life forms' intensely reliant on Plants. Plants assume indispensable job on earth by giving them pabulum to living, wood for houses and different assets to live. Thus, we should deal with plants and farming harvests. About $68 \%$ of the populace relies upon agribusiness. Ranchers have cosmically tremendous scope of assorted variety for winnowing sundry harmonious harvests and finding the apt pesticides for plant. Ailment on plant prompts the vital decrease in both the quality and amount of rural items. The investigations of plant infection allude to the investigations of outwardly clear examples on the plants. Checking of wellbeing and sickness on plant assumes a fundamental job in prosperous development of harvests in the ranch. In early days, the checking and examination of plant maladies were done physically by the skill individual in that field. This requires colossal measure of work and also requires extortionate handling time. Early recognition and exact recognizable proof of sicknesses can control the spread of contamination; it ends up easy to distinguish plant illness with picture preparing procedures. For the most part we can watch the side effects of ailment on leafs, stems, blossoms and so on. So here we use plant leafs for ID of sickness influenced plants. Distinctive plant leaf bears diverse maladies. There are a rundown of techniques and classifiers to distinguish plant leaf illnesses, the examination is made on the plant leaf infection discovery using Digital Image processing approach.
\end{abstract}

Keywords: Plant disease detection, Image Processing, Modified K-means Clustering, Modified Gray-Level Co-Occurrence Matrix(MGLCM ),Modified Support Vector Machine(SVM).

\section{INTRODUCTION}

Digital image process is the utilization of PC calculations to perform picture process on advanced pictures. It allows a far more extensive change of calculations to be connected to the PC record and may keep away from issues like the development of commotion and flag bending all through procedure. Computerized picture process has horrendously vital job in agribusiness field. it's generally acclimated watch the harvest ailment with high precision. Identification and acknowledgment of maladies in plants abuse computerized picture technique is incredibly successful in giving manifestations of trademark sicknesses at its beginning periods. Plant pathologists will break down the computerized pictures abuse advanced picture process for diagnosing of harvest maladies. PC Frameworks zone unit created for agrarian applications, similar to recognition of leaf maladies, organic products sicknesses and so on out and out these procedures, computerized pictures are gathered utilizing a camera and picture process strategies are connected on these photos to separate significant information that are fundamental for examination. The maladies are for the most part on leaves and on stem of plant. The illnesses are viral, bacterial, parasitic, maladies because of creepy crawlies, rust, nematodes and so forth on plant. It is vital errand for ranchers to discover these infections as ahead of schedule as could reasonably be expected. Following model demonstrates that how maladies on cotton plant decreases the efficiency. Picture handling strategies could be connected on different applications as pursues:

Farming has turned out to be much a great deal of than just an approach to nurture consistently developing peoples. Plants have turned into an indispensable wellspring of vitality, and are an essential piece in the riddle to determine the matter of world warming. Infections in plants expands the estimation of rural creation and an absolute monetary fiasco of makers if not relieved suitably at beginning periods. This can affect negative on the nation whose financial pay is exclusively relies upon horticultural produces. Strategies needs to screen their plants routinely and watch any essential manifestations to keep the unfurl of a plant disorder, with minimal effort and spare the significant piece of the generation. In 2015 Ghana lose $30 \%$ of its yearly harvest respects bugs and bug pervasion, the circumstance, was credited to limited access to plant wellbeing administrations because of insufficient expansion officers. Commitment of expert agriculturists may be costly especially in remote confined geographic districts. Customarily most ranchers embraces eye perception as the fundamental strategy for recognizing sicknesses among their harvests and delivers. This methodology ends up tedious when ranch lands turns out to be expansive. However, the interest of this system is constant perception of the division by an individual having predominant information in regards to the plants and its relating ailments. In addition, delegating such an individual would may demonstrate expensive. An elective technique is looking for guidance from the expert by ranchers when indications of infections crop up, and the master exhortation should come in time else it could winds up in misfortune. Conclusion of sickness on plant will conjointly be worn out research facility testing. Be that as it may, this procedure needs tasteful research center conditions on with gifted information. The pathogen discovery ways will offer a great deal of right outcomes.

\section{LITERATURE SURVEY}

The vegetation records from hyper strange information have been appeared to be devious seeing of plant defilements. In any case, they can't see specific illnesses on gather. Wenjiang Huang et al built up the new phantom records for perceiving the winter wheat tainting. They think about three unquestionable bugs (Fine shape, yellow rust 


\section{Available online at www.ijrat.org}

and aphids) in winter wheat for their examination. The most and the least gigantic wavelengths for various infections were removed utilizing Help F tally. The solicitation rightness' of these new records for sound and contaminated leaves with fine advancement, yellow rust and aphids were $86.5 \%, 85.2 \%, 91.6 \%$ and $93.5 \%$ autonomously [1]. Balanced pictures have high check and lucidity than the essential picture. Shading pictures have essential tints red, green and blue. It is hard to understand the applications utilizing RGB by goodness of their range for example 0 to 255 . Thusly they convert the RGB pictures into the diminish pictures. By then the histogram evening out which dissipates the forces of the photographs is related on the picture to improve the plant sickness pictures.

Monica Jhuria et al utilizes picture handling for identification of sickness and the organic product reviewing in [3]. They have utilized fake neural system for identification of sickness. They have made two separate databases, one for the preparation of as of now put away illness pictures and other for the execution of the inquiry pictures. Back spread is utilized for the weight modification of preparing databases. They think about three element vectors, to be specific, shading, surfaces and morphology [3]. They have discovered that the morphological element gives preferable outcome over the other two highlights.

Zulkifli Bin Husin et al, in their paper [4], they caught the stew plant leaf picture and handled to decide the wellbeing status of the bean stew plant. Their system is guaranteeing that the synthetic substances ought to apply to the sick bean stew plant as it were. They utilised the MATLAB for the component extraction and picture acknowledgement. In this paper pre-preparing is finished utilizing the Fourier sifting, edge recognition and morphological tasks. PC vision expands the picture preparing worldview for article characterization. Here computerized camera is utilized for the picture catching and LABVIEW programming device to manufacture the GUI.

The division of leaf picture is vital while extricating the element from that picture. Mrunalini R. Badnakhe, Prashant R. Deshmukh look at the Otsu edge and the kimplies grouping calculation utilized for contaminated leaf examination in [5]. They have presumed that the separated estimations of the highlights are less for kimplies grouping. The clearness of $\mathrm{k}$-implies grouping is more exact than other strategy.

The RGB picture is utilized for the distinguishing proof of malady. Subsequent to applying k-implies grouping strategies, the green pixels is distinguished and afterward utilizing otsu's strategy, shifting edge esteem is gotten. For the element extraction, shading co-event strategy is utilized. RGB picture is changed over into the HSI interpretation. For the surface measurements calculation the SGDM framework is created and utilizing GLCM work the component is determined [6].

The FPGA and DSP based framework is created by Chunxia Zhang, Xiuqing Wang and Xudong Li, for checking and control of plant ailments [7]. The FPGA is utilized to get the field plant picture or video information for checking and conclusion. The DSP TMS320DM642 is utilized to process and encode the video or picture information. The nRF24L01 single chip $2.4 \mathrm{GHz}$ radio transmitter is utilized for information exchange. It has two information pack and transmission strategy to meet client's diverse need and uses multi-channel remote correspondence to bring down the entire framework cost.

Shantanu Phadikar and Jaya Sil utilizes design acknowledgment procedures for the distinguishing proof of rice illness in [9]. This paper depicts a product model for rice illness location dependent on tainted picture of rice plant. They utilized HIS model for division of the picture in the wake of getting the intrigued locale, at that point the limit and spot recognition is done to distinguish contaminated piece of the leaf.

Different creators have utilized the diverse ways to deal with identify the leaf sicknesses for various sorts of plants and yields and so forth. The survey of these considered methodologies is condensed in table 1 with their key highlights.

Sjadojevic et al. [7] have displayed the idea of profound convolution neural system (CNN) and calibrating for the recognizable proof of plant leaf illnesses. Creators have considered thirteen unique kinds of dataset pictures with solid leaf pictures for the experimentation. Profound learning based Caffe system has been utilized alongside the arrangement of loads learned on an extremely extensive dataset by creators. The center of system is created in $\mathrm{C}++$ and gives order line, Python, and MATLAB interfaces. Creators have utilized the 10-overlay cross approval test for the exactness evaluation. The general outcomes demonstrates the exactness of $96 \%$ and accuracy esteem lie between $91 \%$ to $96 \%$.Fine-tuning has not indicated huge changes in the general precision, however enlargement process had more noteworthy impact to accomplish decent outcomes.

Sannakki et al. [12] has utilized feed forward back engendering Neural System based method for the analysis and arrangement of sicknesses in grape leaf. Creator has utilized the pictures of grape leaf with complex foundation for the analysis as information. Further anisotropic dissemination is utilized to expel the commotion of the picture which is additionally portioned utilizing k-implies grouping. At last outcomes are watched utilizing neural system. Results are probed fleece mold and fine buildup pictures with reenactment in MATLAB. Disarray network is considered with the genuine positive and false positive parameters for the approval of results. The creator professed to have the preparation exactness of $100 \%$ whenever utilized tone highlight alone.

\section{DIFFERENT DISEASE TYPES AND SYMPTOMS}

The real classes of plant leaf infections depend on viral, parasitic and microscopic organisms. Here are some basic side effects that ought to be remembered whether plant development appears to be low.

\subsection{Fungal disease symptoms}

Plant leaf maladies, those acknowledged by living being are talked about underneath and appeared in Figure 1. for example Late criticize acknowledged by the improvement. At first it impacts logically arranged leaves that take after, water-doused and spot of diminish green shading. Further ailment sway fabricates, impacts unmistakable leaves moreover and dynamically settled spots propels toward getting the opportunity to be dim. 


\section{E-ISSN: 2321-9637}

\section{Available online at www.ijrat.org}

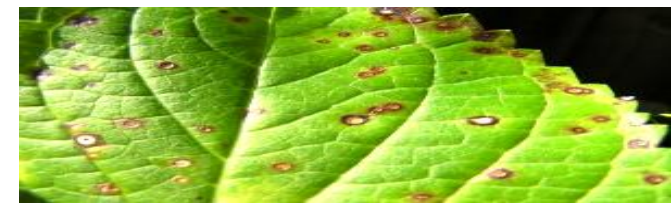

Fig-1: This fungal disease creates circular leaf spots. The spot's center will turn tan or light gray

\subsection{Bacterial disease symptoms}

The malady is described by little light green spots which before long come into view as water-drenched. The sores augment and afterward show up as dry dead spots as appeared in Figure 2.

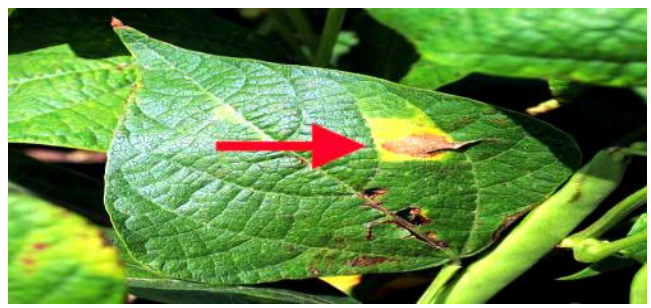

Fig-2: Dark red kidney bean leaf showing bacterial leaf spot symptom

\subsection{Viral disease symptoms}

Among all plant leaf ailments, those brought about by infections are the most hard to analyze. Infections produce no indications that can be promptly watched and regularly effectively mistook for supplement inadequacies and herbicide damage. Aphids, leafhoppers, whiteflies and cucumber bugs creepy crawlies are normal transporters of this malady, for example Mosaic Virus, Look for yellow or green stripes or spots on foliage, as appeared in Figure 3. Leaves may be wrinkled, twisted and development might be hindered

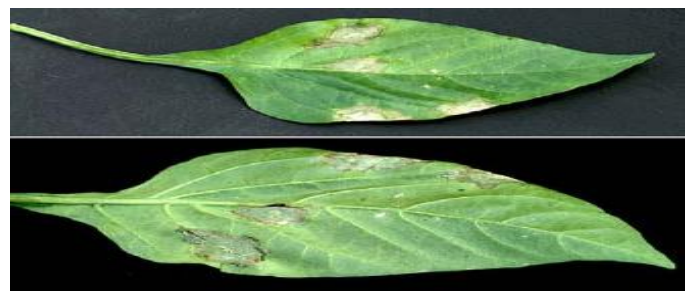

Fig-3 Symptoms of impatiens necrotic spot virus on pepper leaves.

\section{DISEASE CLASSIFICATION STRATEGIES}

Systems on picture handling pestilence in crops produces actual creation and economical misfortunes in farming exchange around the world. perception of wellbeing and location of infections in plants and trees is essential for property farming. To the best of our information, there's no gadget monetarily open for period evaluation of wellbeing conditions in trees. The grouping procedures are frequently observed as augmentations of the recognition techniques, anyway as opposed to endeavoring to watch only one explicit affliction in the midst of very surprising situations. 4.1 Neural Networks

This is the methodology to division of the photos into leaf and foundation inside the accompanying assortment of size and shading choices are extricated from each the RGB and HSI portrayals corresponding to that picture. Those key factors are at long last bolstered to neural systems and connected arithmetic classifiers that are acclimated affirm the plant condition.

\subsection{SVM}

The technique utilizes many shading portrayals all through its execution. The division among leaves and foundation is performed by a MLP neural system, that is including a shading library structured from the earlier by proposes that of an unsupervised self-sorting out guide (SOM). the hues blessing on the leaves are then grouped by recommends that of an unsupervised and unrestrained self-sorting out guide. A hereditary algorithmic program decides the amount of groups to be embraced for each situation. A Help Vector Machine (SVM) at that point isolates bleak and sound areas.

\subsection{Fuzzy classifier}

The strategy endeavors to spot four entirely unexpected natural procedure insufficiencies in plume palm plants. The picture is segmental predictable with shading likenesses, anyway the creators didn't offer any detail on anyway this should be possible. When the division, assortment of shading and surface alternatives are removed and submitted to a fluffy classifier, which, instead of yielding the inadequacies themselves, uncovers the measures of manures that should be acclimated right those insufficiencies..

\section{4 Color analysis}

The strategy plans to locate and separate among four sorts of mineral lacks (nitrogen, phosphorus, potassium and magnesium). The tests were performed exploitation fava bean, pea and yellow lupine leaves. prior to the shading examination, the photos are conceived again to the HSI and $\mathrm{L} * \mathrm{a} * \mathrm{~b} *$ shading zones. the shading varieties between sound leaves and furthermore the leaves underneath investigate at that point affirm the nearness or nonattendance of the insufficiencies. Geometer separations determined in each shading territories measure those varieties.

\section{$4.5 \mathrm{KNN}$}

k-Closest Neighbor could be a simple classifier inside the AI strategies wherever the classification distinguishing proof is accomplished by unmistakable the nearest neighbors to question|a question la question $\}$ models thus construct utilization of these neighbors for assurance of the class of the inquiry. In $\mathrm{KNN}$ the grouping I. e. to that class the given reason for existing is has a place depends on the count of the base separation between the given reason and distinctive focuses. As a classifier the nearest neighbor doesn't typify any instructing strategy. it's not relevant just if there should be an occurrence of tremendous assortment of training precedents since it isn't solid to wheezy data. the hole between the investigate tests and instructing tests is determined for the plant leaf arrangement. amid this technique it discovers comparative measures and thus the class for investigate tests. An example is classed upheld the absolute best assortment of votes from the $\mathrm{k}$ neighbors, with the example being allocated to the classification most regular among its $\mathrm{k}$ closest neighbors. $\mathrm{K}$ could be a positive entire number, by and large small. In the event that $\mathrm{k}=1$, at that point the example is simply allocated to the classification of its closest neighbor.

\section{PROPOSED NOVEL METHODOLOGY}

The essential advance for picture handling is picture procurement pursued by picture pre-preparing for upgrading the complexity of the picture, this improved 
Available online at www.ijrat.org

picture is fragmented by utilizing distinctive picture division calculations like Modified OTSU calculation, Modified K-Means Bunching Algorithm, and so on. Highlights from the picture can be gotten by utilizing Modified Gray-Level Co-Occurrence Matrix (MGLCM) which is a proficient method to remove the highlights from the picture. After extraction of highlights from the picture there is a need for grouping of the malady and indicate the measure of sickness influenced to that comparing leaf, this should be possible by utilizing Modified Support Vector Machine (MSVM).

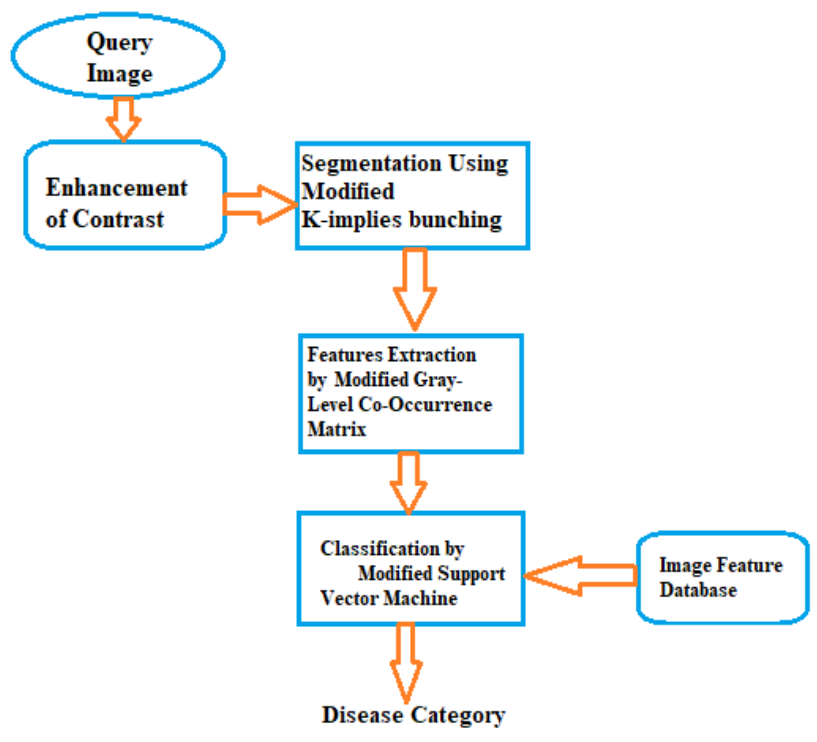

Fig-4 Essential strides for plant sickness recognition And

\subsection{Image Acquisition}

$$
\text { , grouping. }
$$

The data corresponding to the crop leaf are caught via digital camera. This picture is in RGB (Red, Green And Blue) structure. shading change structure for the RGB leaf picture is made, and afterward, a gadget autonomous shading space change for the shading change structure is connected.

\subsection{Image Pre-Processing}

To expel commotion in picture or other item expulsion, diverse pre-handling systems is considered. Picture cutting for example trimming of the leaf picture to get the intrigued picture locale. Picture smoothing is finished utilizing the smoothing channel. Picture upgrade is done for expanding the complexity.

\subsubsection{Low Contrast}

Low Contrast picture is a where Image pixel esteems are focused close to a limited range. Because of this picture looks odd and effectiveness of removing the highlights may get decreased definitely. Along these lines, to show signs of improvement highlights to identify the malady picture ought to be upgraded.

\subsubsection{Contrast Enhancement}

The first picture is the picture given to the framework and the yield of the framework after complexity upgrade is Enhanced Image, the picture in the wake of evacuating the sharp edges is called differentiate Modified.

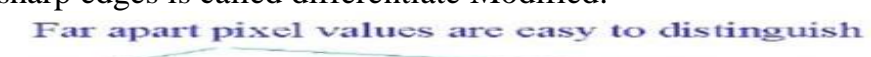

$\mathrm{O}$
Fig-5 Pixel Range

The RGB images into the grey images using color conversion using equation (1).

$f(x)=0.3 * R+0.6501 * \mathrm{G}+0.224 * B$

At that point the histogram evening out which appropriates the forces of the pictures is connected on the picture to upgrade the plant ailment pictures.

\subsection{Image Segmentation}

Segmentation implies dividing of picture into different piece of same highlights or having some likeness. The division should be possible utilizing different strategies like Modified otsu' technique, Modified k-implies grouping, changing over RGB picture into HIS model and so on.

\subsubsection{Modified Otsu Threshold Algorithm}

Thresholding makes double pictures from dark dimension pictures by setting all pixels beneath some edge to zero and all pixels over that limit to one. The Modified Otsu calculation characterized is, as per the following

- According to the edge, Separate pixels into two bunches.

- Then locate the mean of each bunch.

- $\quad$ Square the distinction between the methods.

- Multiply the quantity of pixels in a single bunch times the number in the other.

The tainted leaf demonstrates the indications of the ailment by changing the shade of the leaf. Subsequently the greenness of the leaves can be utilized for the discovery of the tainted part of the leaf. The R, G and B part are removed from the picture. The limit is determined utilizing the Modified Otsu's strategy. At that point the green pixels is covered and expelled if the green pixel powers are not exactly the figured edge.

\subsubsection{Modified K-implies bunching}

The Modified K-implies bunching is utilized for grouping of item dependent on a lot of highlights into $\mathrm{K}$ number of classes. The order of article is finished by limiting the entirety of the squares of the separation between the item and the relating group.

The calculation for Modified $\mathrm{K}$ - implies Clustering:

1. Pick focus of $\mathrm{K}$ bunch, either haphazardly or dependent on some heuristic.

2. Assign every pixel in the picture to the bunch that limits the separation between the pixel and the group focus.

3. Again figure the bunch focuses by averaging the majority of the pixels in the group. Rehash stages 2 and 3 until combination is accomplished.

\subsection{Features Extraction}

Highlight extraction assumes a critical job for distinguishing proof of an article. In numerous use of picture handling highlight extraction is utilized. Shading, surface, morphology, edges and so forth are the highlights which can be utilized in plant ailment identification. In highlight extraction thinks about shading, surface and morphology as a component for illness recognition. They have discovered that morphological outcome gives preferred outcome over different highlights. Surface methods how the shading is dispersed in the picture, the unpleasantness, hardness of the picture. It can likewise be 255 utilized for the identification of contaminated plant regions

\subsubsection{Modified Gray-Level Co-Occurrence Matrix (MGLCM):}


The surface channel capacities give a factual perspective on surface dependent on the picture histogram. These capacities can give valuable data about the surface of a picture however can't give data about shape, i.e., the spatial connections of pixels in a picture. Another factual strategy that considers the spatial relationship of pixels is the dim dimension co-event network (MGLCM), otherwise called the dim dimension spatial reliance lattice. The tool stash gives capacities to make a MGLCM and get factual estimations from it.

\subsection{Classification}

Modified Support Vector Machine (MSVM):

TSVM is a quantifiable learning-based solver. Bona fide is an investigation of frailty. It goes for getting grabbing, settling on choices from a lot of information. From the figure 6. an unmistakable solicitation issue is given in two dimensional information space. The two sorts of model exhibit the photographs of bow framed and oval things. We can draw a line isolating the two classes and different such potential outcomes exist. Obviously figure (b) is perfect organized over figure (an) in light of the route that there is less mistake. Figure (c) might be the ideal line of package. A line advances toward a plane in the event that we have three characteristics factors rather than two, and pushes toward a hyperplane if there are diverse properties.

\section{RESULTS AND DISCUSSION}

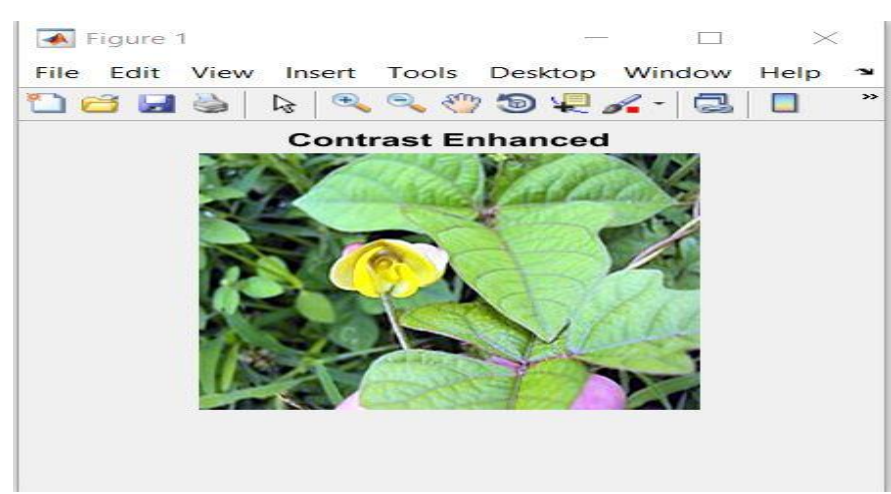

Fig 7: Image of the Black gram leaf

The above figure 7. Shows a healthy leaf of black gram leaf where leaf has no disease affected. Now this healthy leaf is pre-Processed i.e., contrast enhanced of healthy leaf.
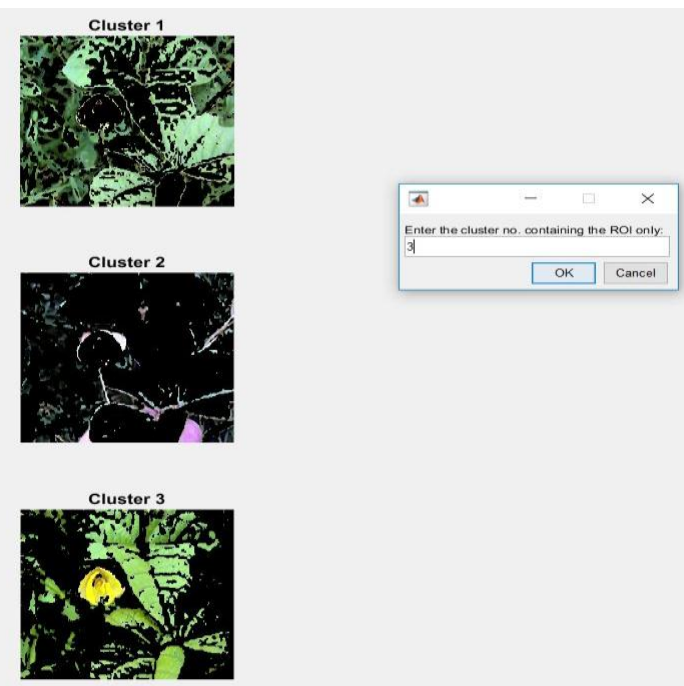

Fig 8: Simulation result after applying the Modified Kmeans algorithm

The figure 8 shows the clusters. Here by using Modified Kmeans segmentation algorithm clustering of pixels were performed. This is the efficient way of clustering discussed in section 5.2.3.

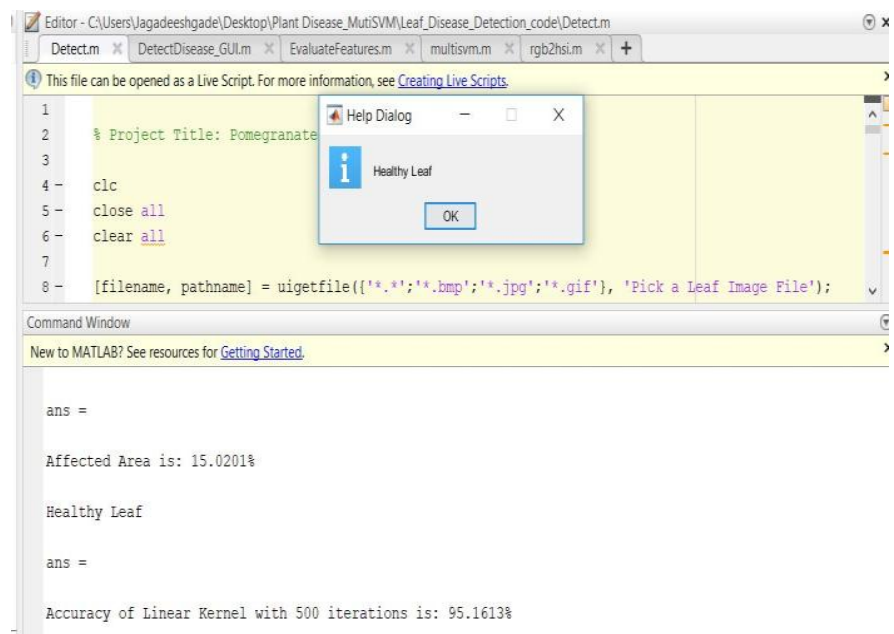

Fig 9: \% of effectiveness in the black gram leaf and condition of leaf

The above figure 9 shows simulation result of healthy leaf, after 500 iterations the leaf is categorized as healthy leaf with the accuracy of $95.1613 \%$ which is highly accurate compared to OTSU algorithm, Here this result is obtained by using the modified K-means clustering algorithm in segmentation and modified SVM for classification of diseases. OTSU algorithm is globally threshold where as modified K-means clustering is 2locally threshold and grouping of clusters can be done by using color images itself. Where as modified otsu will do the analysis by converting the image into gray level image
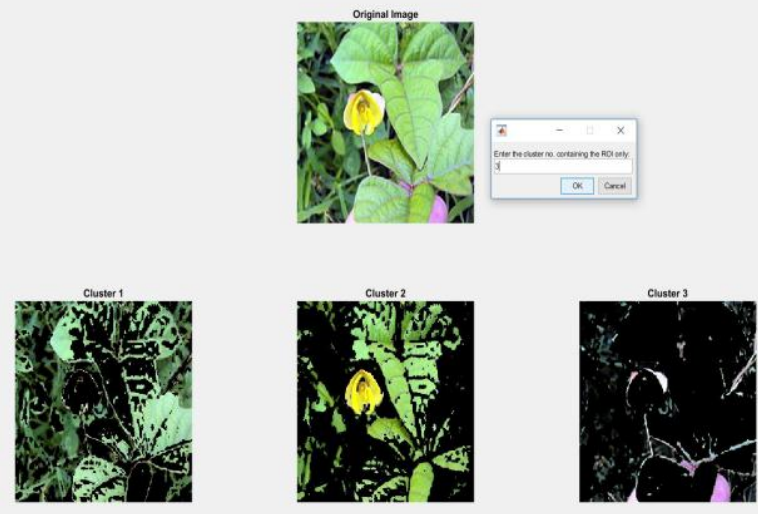

Fig 10: Grouping into Clusters by using GUI MATLAB code

By using Graphical User Interface MATLAB code healthy leaf is subjected to clustering. 


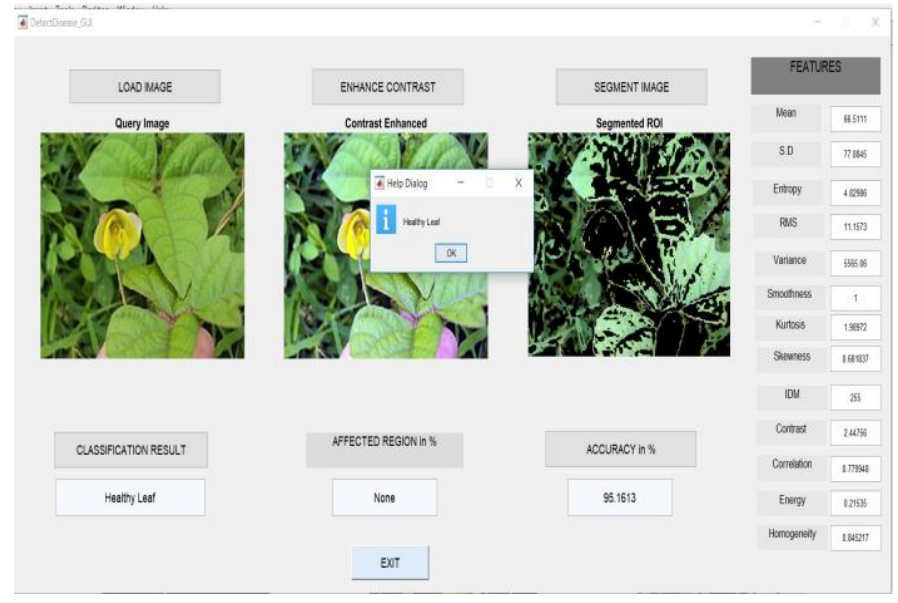

Fig 11: Simulation result by using GUI MATLAB code

MATLAB GUI code gives the efficient page which is the interface face between user and MATLAB code. By using this GUI code one can superss the implementation details and results are displayed on that GUI page where a low man can understand.

The above figure 12. Shows a leaf effected by Bacterial blight disease. Now this bacterial blight leaf is preProcessed i.e, contrast enhanced of healthy leaf

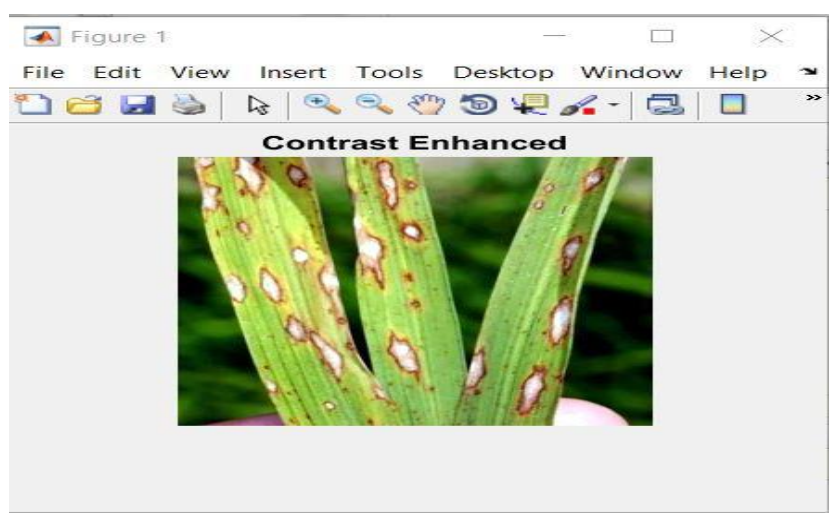

Fig 12: Image of the Paddy leaf

Above figure-12 is the enhanced image of Paddy leaf by using image pre-processing technique. There are few food crops like Paddy, Wheat, ragi, maize etc. Among these crops 77 percent of population in Andhra Pradesh depends on paddy and diseases affected to paddy are very high, we have taken one among the problems and analyzed using smart image processing techniques as shown in figures 13 and 14.
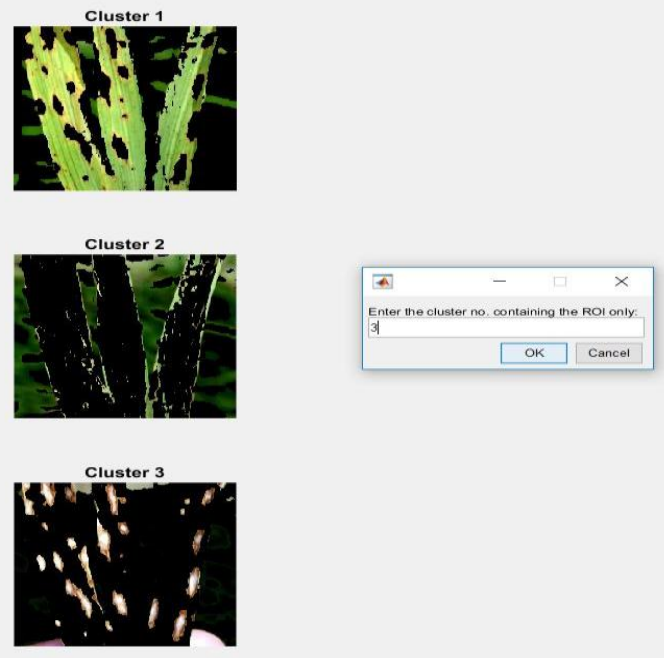

Fig 13: Simulation result of Paddy leaf sample after applying the Modified K-means algorithm

The figure-13 shows the clusters. Here by using K-means segmentation algorithm clustering of pixels were performed. By using this clusters Modified Gray-Level CoOccurrence Matrix(MGLCM ) will extract the features and SVM will classifies the disease .

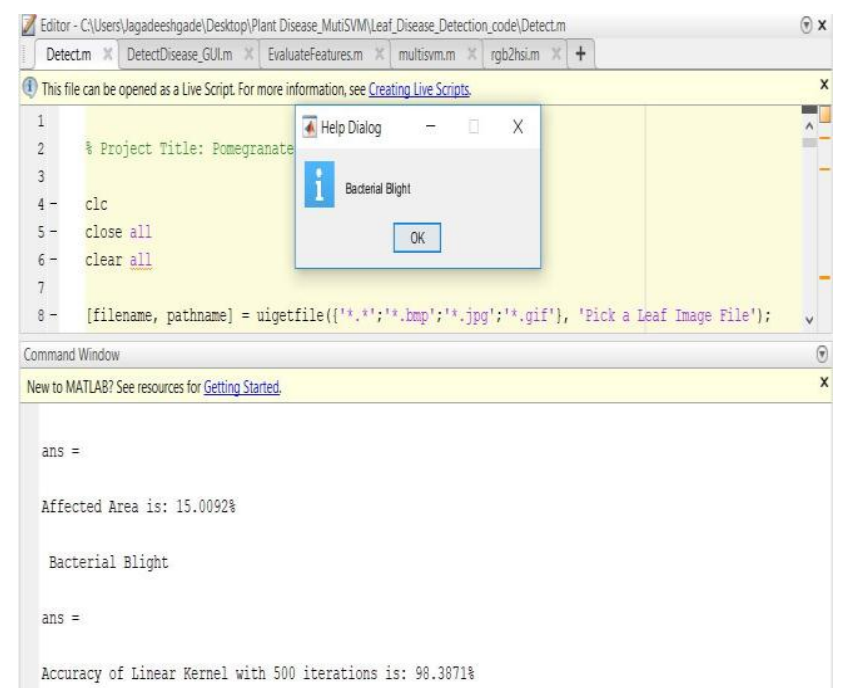

Fig 14: \% of effectiveness in the Paddy leaf sample and type of disease affected to the leaf

Figure-14 clearly gives the name of the disease that affects the paddy leaf: Bacterial Blight. and percentage of affected area on the leaf.

\section{Conclusion and Future Work}

Human life is totally reliant upon nature and plants. In this way, there ought to be uncommon strategies to spare plants from illnesses. The diminishing in yield creation likewise influences the economy of the nation. There is the need of proper research strategy that can consequently recognize the plant leaf ailment. In this examination paper, we have decided the distinctive methodologies utilized by various creators for various sort of plant ailments. The considered idea utilized for characterization are SVM, neural system, hereditary calculation, discriminant work approach, back engendering neural system and so on. The recognized 


\section{Available online at www.ijrat.org}

infections plant are grape, soybean, citrus, grape, [11]. Ramakrishnan, M., and A. Nisha. "Groundnut leaf groundnut and some other accessible dataset with their distinctive sickness types. From these arrangement and identification strategy, there is have to build up some upgraded technique as existing methodologies are not fit for various leaf malady types and on the off chance that any of idea demonstrates proficient outcomes, at that [12] point those outcomes are with constraints

The framework can work broadly by anticipating the sort of plant, the sort of sickness and prescribe the particular pesticides or fungicides to be utilized, henceforth suggested for future work..

\section{Acknowledgments}

Our thanks applause to all-powerful God and all staff individuals from Gudlavalleru Engineering college's electronics and Communication Engineering branch for their different commitment towards this paper.

\section{REFERENCES}

[1]. Arivazhagan, S., R. NewlinShebiah, S. Ananthi, and S. Vishnu Varthini. "Detection of unhealthy region of plant leaves and classification of plant leaf diseases using texture features." Agricultural Engineering International: CIGR Journal 15, no. 1 (2013): 211-217.

[2]. Kranz, J. "Measuring plant disease." In Experimental techniques in plant disease epidemiology, ISSN no. 978-3-642-95534-1, page no. 35-50. Springer Berlin Heidelberg, 1988.

[3]. James, W. Clive. "Assessment of plant diseases and losses." Annual Review of Phytopathology Vol. 12, issue no. 1, page no. 27-48, 1974.

[4]. Khirade, Sachin D., and A. B. Patil. "Plant Disease Detection Using Image Processing." In Computing Communication Control and Automation (ICCUBEA),2015 International Conference on, pp. 768-771. IEEE, 2015.

[5]. Mahlein, Anne-Katrin, Erich-Christian Oerke, Ulrike Steiner, and Heinz-Wilhelm Dehne. "Recent advances in sensing plant diseases for precision crop protection." European Journal of Plant Pathology 133, no. 1 (2012): 197-209.

[6]. Oostendorp, Michael, Walter Kunz, Bob Dietrich, and Theodor Staub. "Induced disease resistance in plants by chemicals." European Journal of Plant Pathology 107, no. 1 (2001): 19-28.

[7]. Sladojevic, Srdjan, Marko Arsenovic, AndrasAnderla, DubravkoCulibrk, and DarkoStefanovic. "Deep Neural Networks Based Recognition of Plant Diseases by Leaf Image Classification." Computational Intelligence and Neuroscience 2016 (2016).

[8]. Naik, M. Ravindra, and Chandra Mohan Reddy Sivappagari. "Plant Leaf and Disease Detection by Using HSVFeatures and SVM Classifier." International Journal of Engineering Science 3794 (2016).

[9]. Mohanty, Sharada P., David P. Hughes, and Marcel Salathé. "Using Deep Learning for Image-Based Plant Disease Detection." Frontiers in Plant Science 7 (2016).

[10]. Bhong, Vijay S., and B. V. Pawar. "Study and Analysis of Cotton Leaf Disease Detection Using Image Processing." International Journal of Advanced Research in Science, Engineering and Technology 3, no. 2 (2016). disease detection and classification by using back probagation algorithm." In Communications and Signal Processing (ICCSP), 2015 International Conference on, ISNB no. 978-1-4799-8081-9, page no. 0964-0968. IEEE, 2015.

2]. Sannakki, Sanjeev S., Vijay S. Rajpurohit, V. B. Nargund, and ParagKulkarni. "Diagnosis and classification of grape leaf diseases using neural networks." In Computing, Communications and Networking Technologies (ICCCNT), 2013 Fourth International Conference on, ISBN no. 978-1-4799- 39268 , page no. 1-5. IEEE, 2013 